\title{
Meta-Analysis of the Related Nutritional Supplements Dimethyl Sulfoxide and Methylsulfonylmethane in the Treatment of Osteoarthritis of the Knee
}

\author{
Sarah Brien, ${ }^{1}$ Phil Prescott, ${ }^{2}$ and George Lewith ${ }^{1}$ \\ ${ }^{1}$ Department of Primary Medical Care, University of Southampton, Aldermoor Health Centre, Aldermoor Close, Southampton, \\ Hampshire, SO16 5ST, UK \\ ${ }^{2}$ School of Mathematics, University of Southampton, Southampton, Hampshire, SO16 5ST, UK
}

Correspondence should be addressed to Sarah Brien, s.brien@southampton.ac.uk

Received 24 October 2008; Accepted 23 April 2009

Copyright (c) 2011 Sarah Brien et al. This is an open access article distributed under the Creative Commons Attribution License, which permits unrestricted use, distribution, and reproduction in any medium, provided the original work is properly cited.

Dimethyl sulphoxide and methylsulfonylmethane are two related nutritional supplements used for symptomatic relief of osteoarthritis (OA). We conducted a meta-analysis to evaluate their efficacy in reducing pain associated with OA. Randomized or quasi-randomized controlled trials (RCTs), identified by systematic electronic searches, citation tracking and searches of clinical trial registries, assessing these supplements in osteoarthritis of any joint were considered for inclusion. Meta-analysis, based on difference in mean pain related outcomes between treatment and comparator groups, was carried out based on a random effect model. Seven potential trials were identified of which three RCTs, two DMSO and one MSM (total $N=326$ patients) were eligible for inclusion. All three trials were considered high methodological quality. A significant degree of heterogeneity $\left(\chi^{2}=6.28\right.$, $P=.043$ ) was revealed. Two studies demonstrated statistically significant (but not clinically relevant) reduction in pain compared with controls; with one showing no group difference. The meta-analysis confirmed a non significant reduction of pain on visual analogue scale of $6.34 \mathrm{~mm}(\mathrm{SE}=3.49,95 \% \mathrm{CI},-0.49,13.17)$. The overall effect size of 1.82 was neither statistically nor clinically significant. Current evidence suggests DMSO and MSM are not clinically effective in the reduction of pain in the treatment of OA. No definitive conclusions can currently be drawn from the data due to the mixed findings and the use of inadequate dosing periods.

\section{Introduction}

Osteoarthritis (OA) is the most common of all joint disorders and affects over 30 million people in the US and 1 in 10 people aged $35-75$ in the UK [1] and is associated with pain and functional disability, which in turn leads to reduced quality of life and increased risk of further morbidity and mortality [2]. The treatment of OA is largely symptomatic and includes analgesics, NSAIDs as well as exercise and surgical intervention [3]. The long-term use of NSAIDs is associated with serious gastrointestinal side effects [4,5] including 12000 hospital admissions and $\sim 2000$ deaths in the UK every year [6]. Patients with OA turn to complementary and alternative medicine (CAM) to gain symptomatic relief and avoid iatrogenic illness with OA being the sixth most common condition treated with CAM [7]; OA patients use of CAM is substantially greater that in the general population with a reported prevalence of up to $90 \%[8,9]$.

Two nutritional supplements, dimethyl sulfoxide (DMSO, an organic form of sulfur commercially prepared from lignin) and its oxidized form, methylsulfonylmethane (MSM, occurring in green plants fruits and vegetables) have been used to treat arthritic conditions [10]. DMSO is converted in the body to MSM and as MSM remains in the body for longer than DMSO [11], it is suggested that many of the beneficial effects of DMSO are due to the long lasting fraction of DMSO which is converted to MSM [12]. Both have similar pharmacological properties and their putative effects and mechanisms have been reviewed previously (MSM [13-15]; DMSO [16-19]; both [20, 21]). MSM and DMSO have been reported to reduce peripheral pain [22-24], inflammation [25] and arthritis [26], and might inhibit the degenerative changes occurring in OA [27]. 
These compounds may act through their ability to stabilize cell membranes, slow or stop leakage from injured cells and scavenge hydroxyl free radicals which trigger inflammation $[23,25,28-33]$. Their sulfur content may also rectify dietary deficiencies of sulfur improving cartilage formation [34, 35].

DMSO is a topical agent, diluted for therapeutic use (concentrations are expressed \%(v/v)). It penetrates the skin and it is also used as a carrier to aid penetration of other medications $[24,28,36]$. Clinicians are advised to prescribe DMSO for OA for at least three months to ensure a clinical effect. However the optimum dosage for this supplement in OA has not been clearly evaluated as no dose ranging studies have been conducted. Previous empirical reports suggest that the therapeutic concentrations of DMSO are $60 \%-90 \%$ $[18,37]$ and that doses under $10 \%$ are probably clinically inactive [37-39]. There is limited formal safety data and no long-term assessment of DMSO although the toxicity of oral DMSO appears very low (LD50 $=14.5 \mathrm{~g} / \mathrm{kg}$ body weight). Adverse effects associated with topical DMSO administration have been reported (GI upset, skin irritation, and garlic like taste, breath and body odour) [40, 41]. Its garlic odour can compromise blinding in double blinded trials.

MSM is used orally and topically. Like DMSO, the treatment duration for $\mathrm{OA}$ is at least three months. The optimum dosage has not been clearly defined as no dose ranging studies have been carried out. The suggested oral therapeutic doses is $4-6 \mathrm{~g}$ per day [42, 43], although doses of up to $20 \mathrm{~g} /$ day have also been used [44]; over the counter preparations are typically $1-5 \mathrm{~g}$ daily [45]. There is limited formal safety data and no long term assessment. However, MSM is rapidly excreted from the body [46, 47] and animal toxicity studies of MSM showed only minor adverse events using doses of $1.5 \mathrm{~g} / \mathrm{kg}$ and $2.0 \mathrm{~g} / \mathrm{kg}$ of MSM for 90 days. This dose represents a human dose of 105-140 g/day, which is equivalent to 17-23 times the proposed maximum recommended human dose of $6 \mathrm{~g} /$ day [48]. A further study confirmed MSM had no toxic effects on either pregnant rats or their foetus [49]. Only minor adverse effects are associated with MSM administration in humans and include allergy, GI upsets and skin rashes [50].

Two systematic reviews have also been conducted on these supplements. Ameye and Chee [13] conducted a systematic review of a range neutriceuticals in OA which included MSM and concluded that MSM showed "moderate" evidence of efficacy. Brien et al. [21] reported that current evidence precludes definitive conclusions about the efficacy of either supplement but recommended further investigation addressing methodological concerns including optimal dosage and treatment duration. A meta-analysis to assess the most precise estimate of the efficacy of these supplements is therefore timely given their frequent use $[8,9]$ and pertinent because of the withdrawal of some COX-2 inhibitors [5].

\section{Methods}

2.1. Literature Search Strategy. Searches were performed using the following electronic databases to identify relevant
TABle 1: Search strategy for DMSO.

(1) Explode "osteoarthritis"/all subheadings

(2) Explode "degenerative arthritis"/all subheadings

(3) Osteoarthr\$

(4) (1)-(3)

(5) Dimethyl sulfoxide

(6) DMSO

(7) (5)-(6)

(8) Clinical trial

(9) Double blind

(10) Single blind

(11) RCT

(12) Placebo

(13) Randomised

(14) Comparative study

(15) Evaluation study

(16) Control

(17) 8 OR 9 OR 10 OR 11 OR 12 OR 13 OR 14 OR 15

(18) 4 AND 7 AND 17

(19) LIMIT 18 to HUMAN

studies available for inclusion in the meta-analysis; Cochrane Library (1970-2008), MEDLINE (1950-2008), EMBASE (1980-2008), AMED (1985-2008), CINAHL (19822008), SCOPUS (1996-2008) and the National Library for Health (Complementary and Alternative Medicine Specialist Library). Free text searches were performed on each database with the following keywords: osteoarthritis, degenerative joint disorder, dimethyl sulfoxide, DMSO, methylsulfonylmethane, MSM, clinical trial: double blind; single blind; RCT; placebo; randomized; comparative study; evaluation study; control. The search strategies for dimethyl sulfoxide and methylsulfonylmethane are shown in Tables 1 and 2 , respectively.

Citation tracking was undertaken to identify unpublished trials. As numerous pharmaceutical companies market DMSO, it was impractical to contact each of them for unpublished data. Finally we additionally searched four clinical trials registries (http://www.clinicaltrials.gov/; http://www.controlled-trials.com/; http://www.actr.org.au/; and http://www.umin.ac.jp/ctr/), to identify ongoing trials. The last update of searches was performed in June 2008.

2.2. Trial Selection. All articles that reported randomized or quasi-randomized controlled trials (RCTs) comparing oral or topical formulations of DMSO or MSM in the treatment of OA were identified. As current data shows that using greater than 10\% DMSO [35-37] and over $1 \mathrm{~g} /$ day MSM [45] are needed for therapeutic effects only RCTs assessing at least these minimal levels were included. RCTs were included if they were; in humans; reported comparison of DMSO or MSM to placebo; used validated outcome measures for OA; and did not include patients with other joint pathology. 
TABLE 2: Search strategy for MSM.

(1) Explode "osteoarthritis"/all subheadings

(2) Explode "degenerative arthritis"/all subheadings

(3) Osteoarthr\$

(4) (1)-(3)

(5) Methylsulfonylmethane

(6) MSM

(7) (5)-(6)

(8) Clinical trial

(9) Double blind

(10) Single blind

(11) RCT

(12) Placebo

(13) Randomised

(14) Comparative study

(15) Evaluation study

(16) Control

(17) 8 OR 9 OR 10 OR 11 OR 12 OR 13 OR 14 OR 15

(18) 4 AND 7 AND 17

(19) LIMIT 18 to HUMAN

2.3. Data Extraction. Data was extracted independently by two of the authors (S.B. and P.P.). Data regarding publication status, trial design, patient characteristics, treatment regimens, outcome measures, results and findings were extracted. Effect sizes were calculated where appropriate. Where information was insufficient the authors were contacted to request missing information. Disagreements were resolved by discussion by all three reviewers and subsequent consensus.

2.4. Quality Assessment. Two reviewers (S.B. and P.P.) independently assessed the trial quality using the JADAD [51] scale to assess randomization, blinding and withdrawal. The total JADAD score is 5 and studies were considered low quality if they reported a score less than 3, or high quality for scores 3-5. Additional quality assessment was also conducted including assessment of concealment of treatment allocation. Assessment of internal and external validity for these papers have also been reported elsewhere [21]. Disagreements were resolved by discussion with the third author.

2.5. Data Synthesis. The selected outcome measure for analysis was the measurement of pain, as this is the most patient relevant outcome in this condition. The primary outcome measure was mean change in pain from baseline. Pain levels were assessed using either a $100 \mathrm{~mm}$ visual analogues scales (VAS) and on the Western Ontario MacMaster Osteoarthritis Index (WOMAC) pain subscale [52]. As the WOMAC subscales have a 0-20 score, these are adjusted up to a 0-100 range for comparability. These were used to assess the differences between the intervention groups (MSM or DMSO) and the control groups (placebo or standard conventional treatment, diclofenac). Data points were chosen at the end of the treatment period.
2.6. Statistical Analysis. Summary estimates of the treatment effect were calculated using the weighted means of the within study treatment effects [53]. Weighted mean differences and $95 \%$ confidence intervals (CI) were calculated using both a fixed and random effects model. Where data were insufficient the original authors were contacted to request the missing information. Sensitivity analyses were conducted where appropriate. Effect sizes were calculated by dividing the difference in the changes, from baseline to the end of the trial period, between treatment groups by the estimated standard error. An effect size of about 0.8 is considered minimally clinically relevant [54]. Chi square test was used to investigate the heterogeneity in the treatment difference parameter across the studies. Publication bias was explored by using a funnel plot, whereby effect estimates of the pain were plotted against trial sample size. The funnel plot was examined visually but due to the small number of studies it was not feasible to statistically test for symmetry.

\section{Results}

3.1. Three RCTs Met Inclusion Criteria. A total of seven citations were retrieved from the databases that met the inclusion criteria [36, 42, 43, 55-58]. Two further clinical trials were identified from citation tracking $[39,59]$. No further studies were identified from searching clinical trials registries. Citations were excluded if: they were not randomized placebo controlled trials; they reported an intervention other than DMSO/MSM; assessed another treatment condition; or they did not assess either DMSO or MSM.

Seven double blind, placebo controlled, randomized trials, assessing either DMSO or MSM in the treatment of $\mathrm{OA}$ in our literature search which met the inclusion criteria were identified for possible inclusion $[36,39,42,43,56$, 57, 59]. Of these, three were eligible for inclusion in the meta-analysis. Four trials were excluded because either (i) DMSO was not specifically assessed as a therapeutic agent $[57,59]$; (ii) there was incomplete data [42] (mean and SD for placebo group were not reported in the text and attempts to contact the authors were unsuccessful); or (iii) the pain scale was assessed using a Likert scale rather than VAS [39] and hence continuous data could not be extracted for the analysis. Only selected data from one study [36] were included; this study had three intervention arms (diclofenac versus DMSO versus placebo) so only the arms relating to the assessment of DMSO versus placebo were included. A search of trial registries yielded no ongoing trials. Therefore the final analysis included three studies, one of which assessed MSM $[43]$ and two DMSO $[36,56]$.

3.2. Description of Studies. Table 3 describes the main study characteristics of the studies included in the meta-analysis. Further study details relating to the studies included in this meta-analysis can be obtained from our recent review of all clinical trials assessing DMSO/MSM in OA [21]. Overall, the trials allocated 326 patients of which 161 received active treatment (DMSO, $N=136$ or MSM, $N=25$ ). All three trials assessed patients with $\mathrm{OA}$ of the knee 


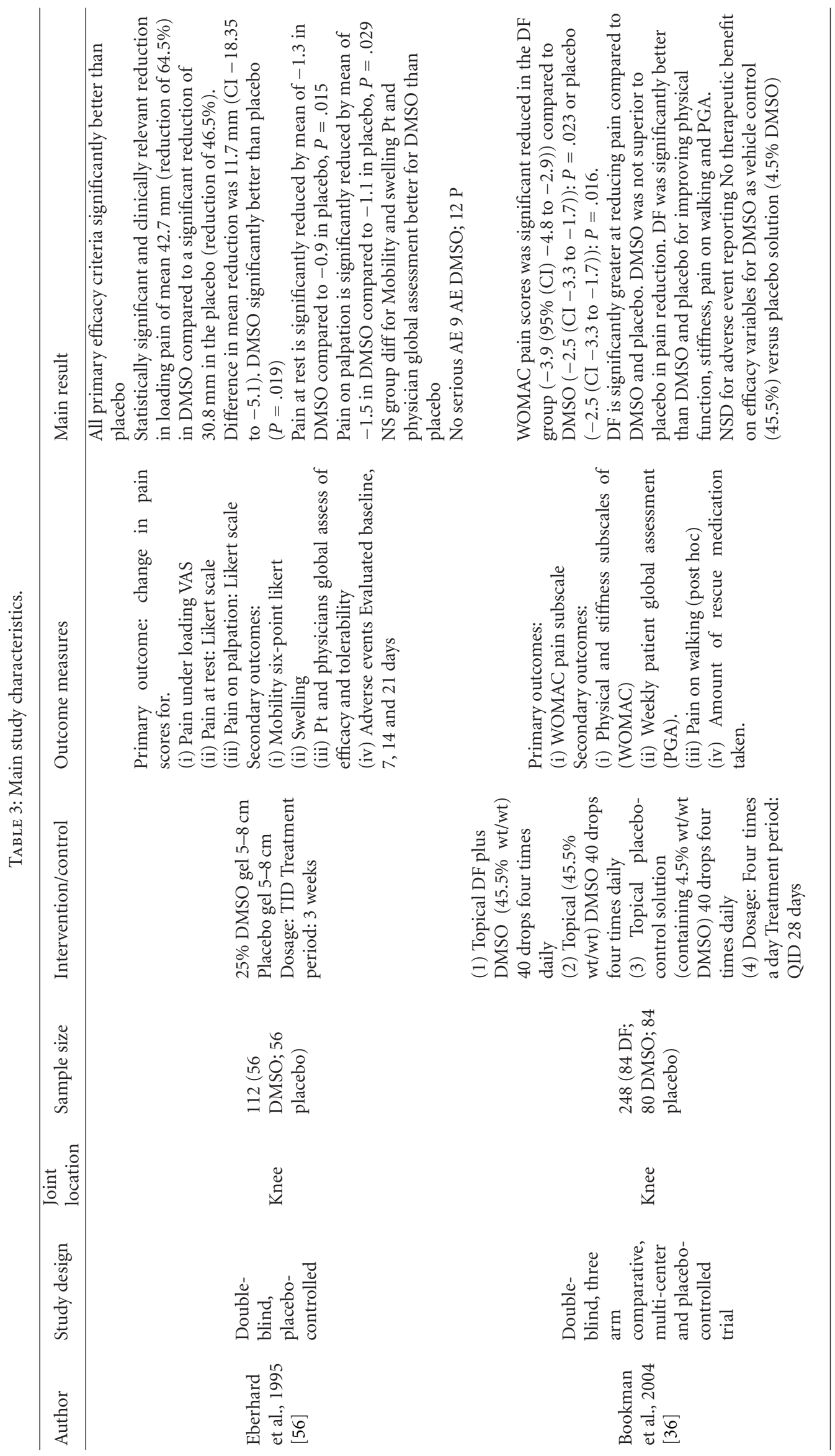




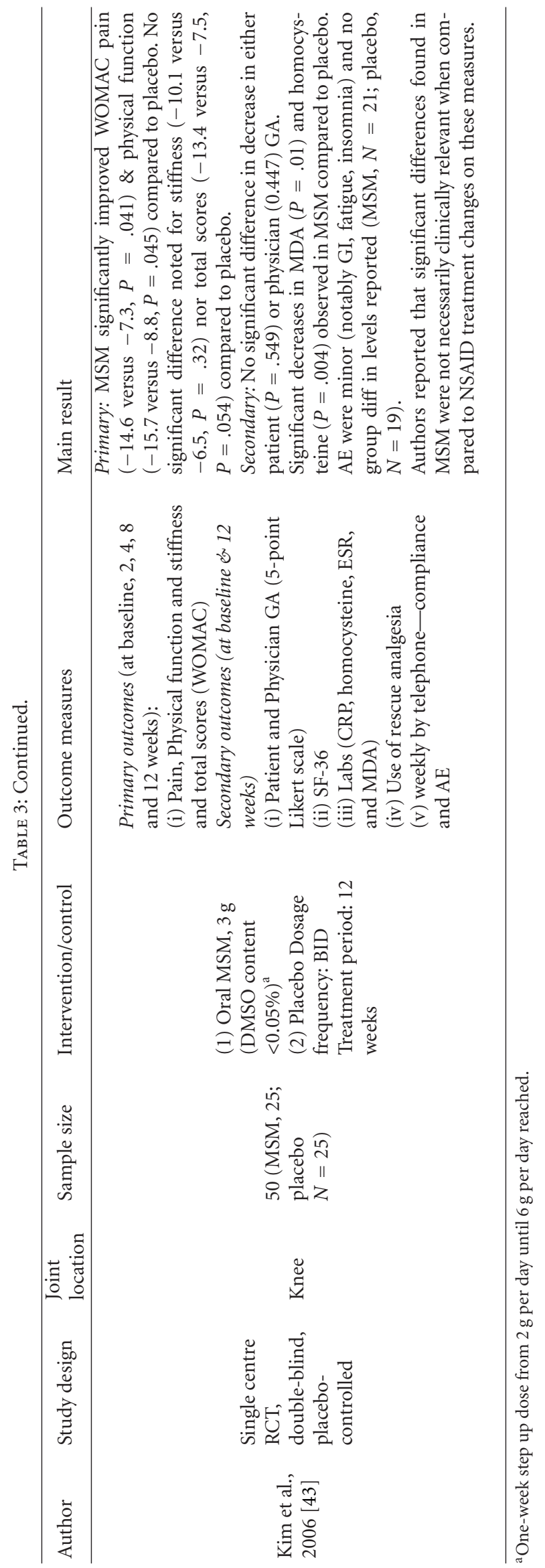


joint. Two trials assessed the supplement DMSO topically administered $[36,56]$ and one MSM, oral administration [43]. All trials assessed these supplements as an alternative rather than adjunctive treatment for $\mathrm{OA}$ of the knee, and rescue medication was allowed. The average mean age of the patients included in these trials ranged from 56 [43] to 62 years $[36,56]$.

3.2.1. Baseline Characteristics. Baseline demographic characteristics were reported in all studies and all showed no significant differences between groups. However, the data describing the characteristics of the patients entered into these three trials was not fully reported by the trials. The average duration of OA was reported in only the Kim study (mean duration of arthritis was 6 years); although patients were included in the Bookman and Eberhardt studies only if they had radiological diagnosed OA for a minimum of 6 months or 5 years, respectively. The severity of OA of included patients was reported in only one trial [43] which stated $95 \%$ of patients entered had low to moderate OA (i.e., Kellegren-Lawrence grades between 0 and 2).

3.2.2. Study Medication. All studies assessed the supplements as alternative treatments with all concurrent systemic and topical medications stopped prior to commencement of the study. The use of rescue medication was reported in two of the studies $[36,43]$. The dosage and treatment duration of the supplements in these trials has been previously reviewed and highlighted concerns about inadequate treatment duration and dosage of these supplements assessed in clinical trials [21]. The doses employed by all three trials assessed in this meta-analysis were all below current recommendations. Duration of treatment was also inadequate in two (the DMSO trials $[36,56])$ of the three trials; the Kim trial assessed MSM for an adequate duration. Compliance assessment was reported in two of the studies ([43] pill count; [36] weighing medication bottles). No follow up assessment was performed in any of the three trials.

3.2.3. Pain as the Primary Outcome. Pain was assessed as the primary efficacy outcome in all these trials. Pain was assessed either by $0-100 \mathrm{~mm}$ VAS [56] or the WOMAC pain subscale $[36,43]$. Those studies reporting pain via the WOMAC pain scales either assessed this using or $0-100$ scale [43] or a $0-20$ scales [36]; the latter results were scaled up to a $0-100$ range for comparability.

The Eberhardt study [56], although it included more patients than the other studies, did not provide any $a$ priori power calculations. However, the observed difference between treatment groups in reduction of pain from baseline to end of treatment of $11.7 \mathrm{~mm}$ was shown to be statistically significant, $P=.0019$; and our sensitivity analysis confirmed power was adequate, that is, greater than $90 \%$. Bookman et al. [36] calculated that 50 patients per group, including allowing for dropouts, would be required for an $80 \%$ power to detect a specified difference between the two treatment arms and a previous analysis confirmed power was adequate [21]. Kim et al. [43] included a power calculation for the improvement from baseline to 12 weeks, claiming that 25 patients per group would be sufficient to detect with $80 \%$ power an improvement in pain score of $25 \%$; however this was not based on the difference between treatment groups. Our subsequent sensitivity analysis confirmed this study had a power of $55 \%$ to detect a difference between treatment groups at $5 \%$ level of significance.

3.2.4. Trial Quality Was High. Table 4 reports the methodological quality characteristics of the included trials. All three trials were of high quality, scoring JADAD scores of either $4[56]$ or $5[36,43]$. All trials were reported as being randomized. The allocation sequence was reported as being adequately generated in two of the trials [36, 43] but no details were provided in the Eberhardt study regarding the randomization process [56]. All the trials were adequately double blinded with the reported use of identical dummy tablets (MSM) or dummy gel (DMSO). They all described withdrawals and exclusions and analyzed all randomly assigned patients and hence conducted an intention to treat. Two of the trials $[36,56]$ reported their approach for handling missing data; using last observation carried forward for imputations [60].

3.2.5. Meta-Analysis_-Effect on Joint Pain. Key data from the included trials is presented in Table 5. Two of the trials [43, 56] reported mean differences that favour DMSO/MSM over placebo with effect sizes of 3.53 and 2.06, respectively; and both trials show 95\% CI that do not overlap zero indicating significant differences. Bookman et al. [36] identified no group differences in pain reduction ( effect size $=0.0$ ). The initial fixed effects meta-analysis of all the trials indicates a statistically significant reduction of pain scores of $6.3 \mathrm{~mm}$ (CI: $2.41,10.27$ ) from baseline in favour of DMSO/MSM compared to placebo (Figure 1). However this change is not clinically significant; a significant improvement of 9$12 \mathrm{~mm}$ on WOMAC subscales in pain, is considered clinically relevant [61-63] as is a $>17.5 \mathrm{~mm}$ change [64] in VAS pain scales.

A chi-squared test of homogeneity was just significant $\left(\chi^{2}=6.28, P=.043\right)$, suggesting that the effects are more variable than would be expected by chance and indicating inconsistencies in the results of the studies. A random effects meta-analysis identified a slight increase in SE of the mean difference of $6.3 \mathrm{~mm}$ with $\mathrm{SE}=3.49 \mathrm{~S}$ and $95 \% \mathrm{CI},(-0.49$ to 13.17) which is not quite statistically significant. The funnel plot also confirmed asymmetry in the results, due to the Bookman et al. study [36] to the left of the common effect indicating the possibility of publication bias.

\section{Discussion}

The findings of this meta-analysis suggest that based on available evidence, DMSO/MSM leads to an overall reduction in pain VAS of $6.34 \mathrm{~mm}$ compared to placebo. This is not statistically significantly superior to placebo in reducing pain associated with OA of the knee. Results from the Bookman trial [36] showed no difference in effect between treatment 


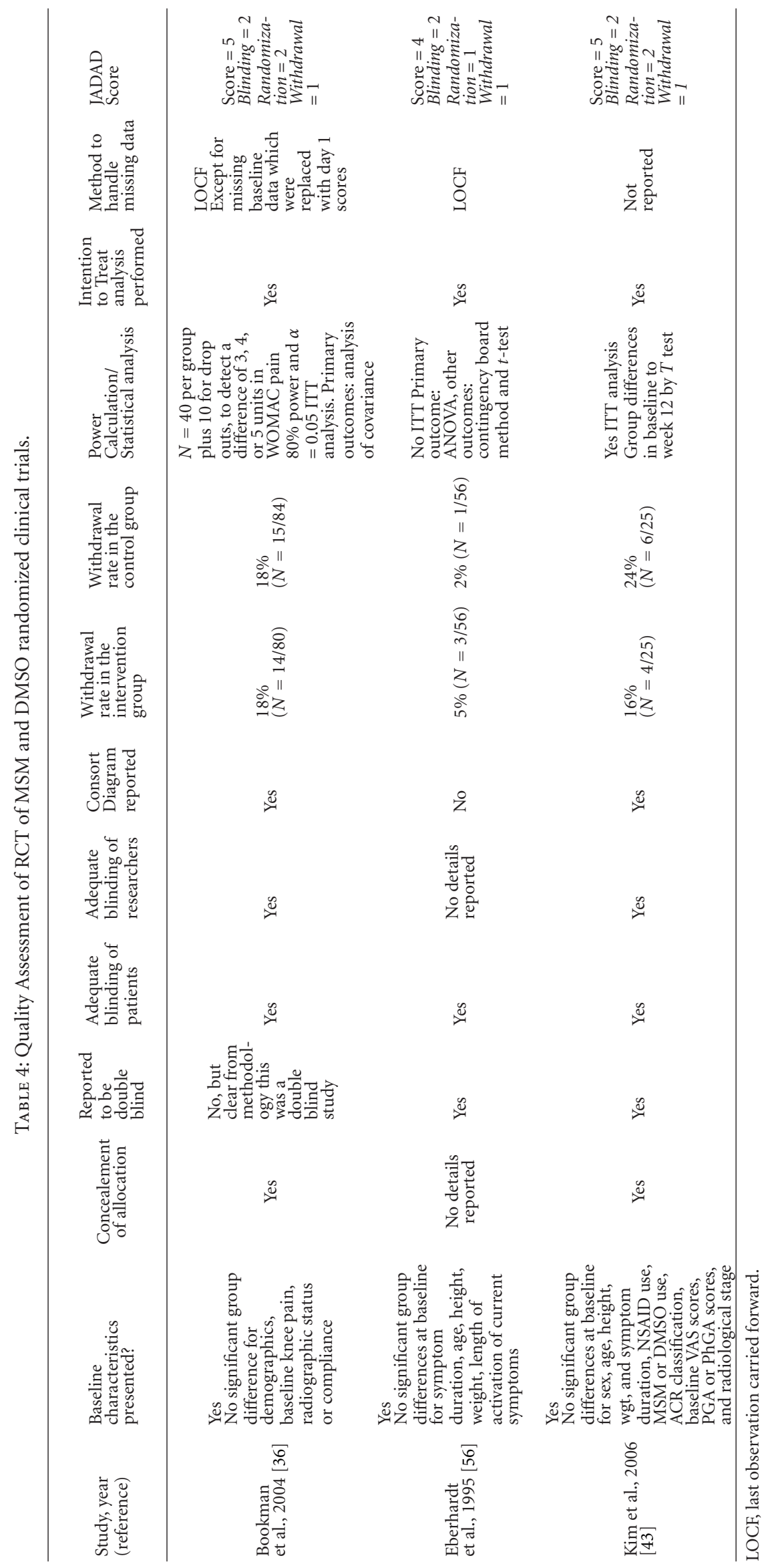




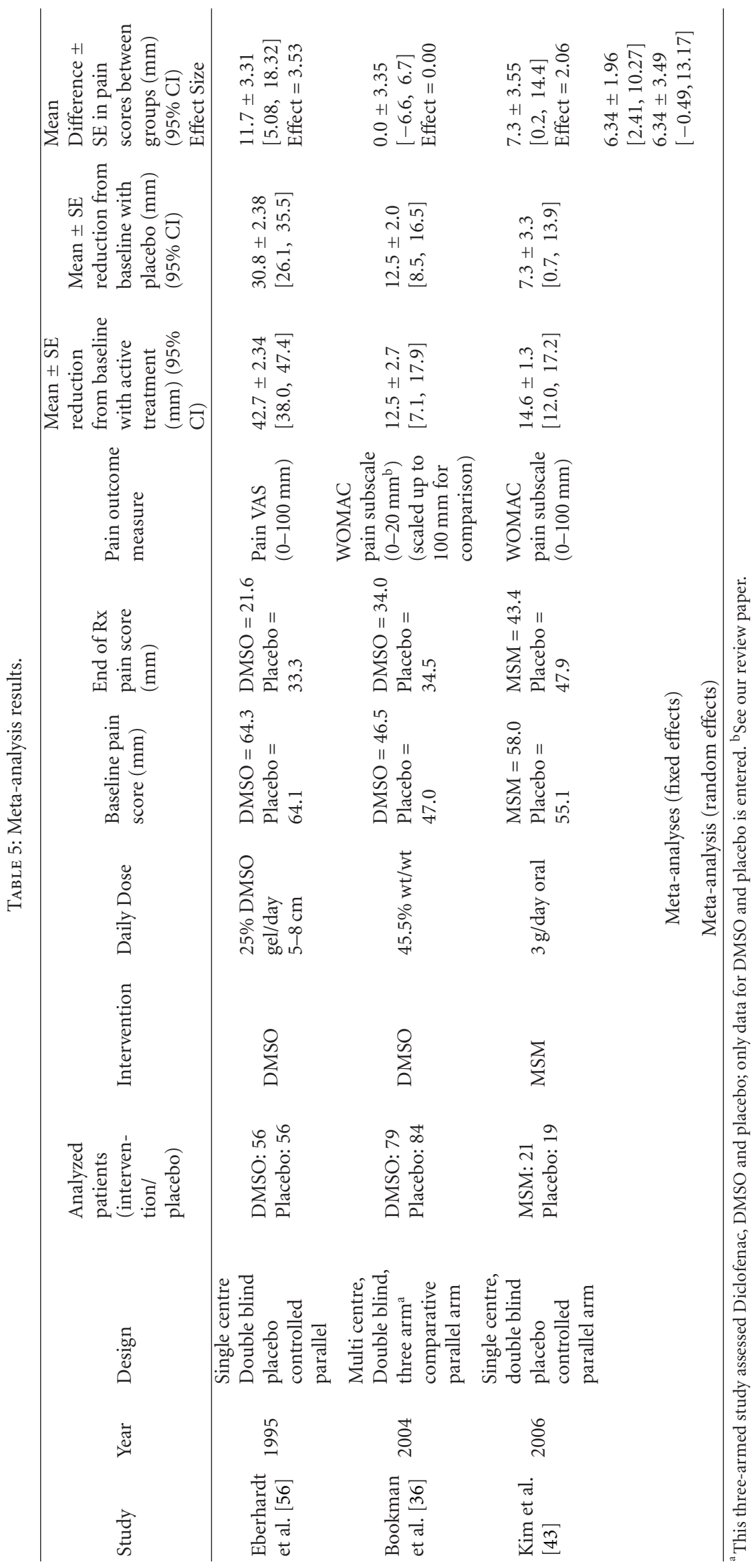




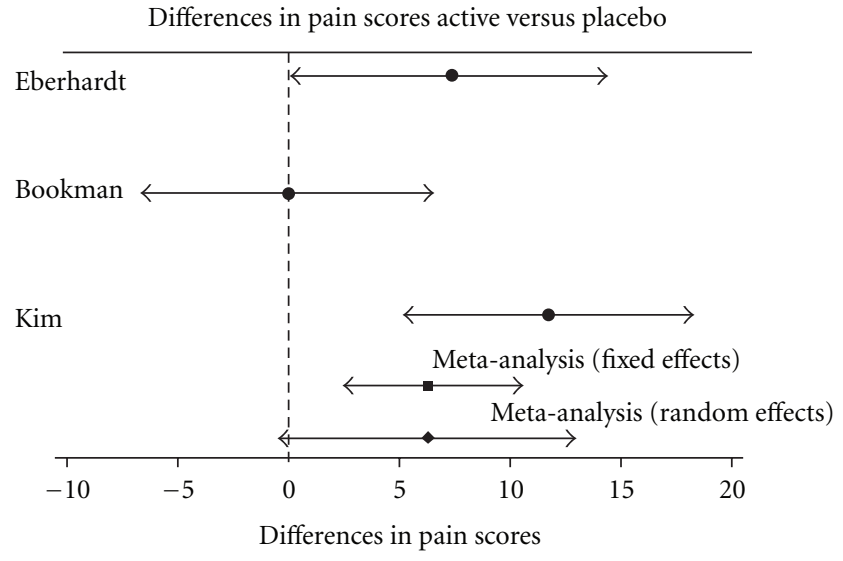

FIGURE 1: Meta-analyses of randomized controlled trials.

and placebo which is in contrast to the results from the other two trials; this may be due to a larger than anticipated placebo response or may reflect possible publication bias.

Clinical significance is critical for meaningful metaanalyses [65] designed to assist clinicians in their decision making. Current guidelines suggest a minimal clinically important improvement (MCII) for the pain subscale for WOMAC in patients with knee OA requires a minimum change of $19.9 \mathrm{~mm}$ from baseline to end of treatment [61]; whilst the MCII for VAS of pain in this condition is a minimum of $17.5 \mathrm{~mm}$ [64]. It has also been observed that baseline pain levels affect patient perceived clinical significance. Recommendations have been proposed for future trials that are based on reporting MCII specific to baseline severity levels [61]. Pain score reductions from baseline to end of treatment, in those receiving active treatment with DMSO were $12.5 \mathrm{~mm}$ ([36]; WOMAC pain subscale) and $42.7 \mathrm{~mm}$ ([56]; VAS); and $14.6 \mathrm{~mm}$ ([43]; WOMAC pain subscale) for patients receiving MSM. Based on current guidelines, only those patients in the Eberhardt study (both active and placebo treated) reported clinically meaningful reductions in their pain and showed a reduction in pain levels comparable with standard conventional treatment with COX 2 inhibitors such as Rofecoxib (35.4 mm decrease [66]; $28.1 \mathrm{~mm}$ [67]). This meta-analyses confirms that based on the current limited evidence these nutritional supplements are neither statistically nor clinically more effective than placebo in reducing pain in knee OA.

4.1. Strengths and Limitations. Only randomized, double blind, placebo controlled trials were included in this investigation. Our review is based on a systematic and thorough literature search. It is unlikely we missed relevant trials data but as possible data was not sought from pharmaceutical companies this cannot be ruled out. Data extraction, including quality assessment, was completed independently by two authors to minimize bias. The reporting quality of the trials was acceptable and the quality assessment tool is validated and its components are associated with bias. All three studies assessed OA of the knee hence enabling direct comparison between trials.
There are some limitations of this meta-analysis. First, only three trials were available for inclusion assessing $N=$ 326 patients in total, of which $N=161$ received active treatment. Further trials may therefore affect the future assessment of these supplements. In addition specific methodological concerns have been identified in these trials including the issue of adequate treatment period with only one study assessing the supplements for a clinically relevant time [43].

4.2. Implications for Research and Clinical Practice. Recent reviews ([21] DMSO and MSM; [13] MSM)) of these supplements for OA suggest further studies of these nutritional supplements may be warranted if the study design takes into account the methodological issues identified in this article. This meta-analysis does not currently provide robust evidence to support the use of DMSO or MSM for OA however the conclusions are affected by the number and quality of included trials. The three trials assessed had good methodological quality and adequate reporting yet significant methodological concerns about dosing and treatment duration temper any definitive conclusions. In addition, due to the paucity of studies of these supplements, the publication of further trials may have a major effect on these recommendations.

If we wish to further evaluate these supplements then large scale trials are needed that are adequately powered and assess patients for a clinically relevant duration using optimal dosage. Future trials should also report the minimal clinically important improvement with reference to the patient's baseline severity [61]. A search of clinical trial registries revealed no ongoing trials and it seems unlikely that suitable evidence investigating the effectiveness of these supplements will become available in the near future. The implications for current clinical practice are that these supplements are not specifically effective in decreasing the pain associated with OA knee. Given the additional lack of safety reporting data as previously described in a recent systematic review [21], it is also not possible to have clarity about their safety.

\section{Funding}

Southampton Complementary Medicine Charitable Research Trust (to S.B.); Rufford Maurice Laing Foundation (to G.L.).

\section{Acknowledgments}

The authors thank medical students Nadia Bashir and Henry Lewith for their assistance with data collection.

\section{References}

[1] O. Sangha, "Epidemiology of rheumatic diseases," Rheumatology, vol. 39, supplement 2, pp. 3-12, 2000.

[2] K. M. Jordan, N. K. Arden, M. Doherty et al., "EULAR recommendations 2003: an evidence based approach to the management of knee osteoarthritis: report of a Task Force 
of the Standing Committee for International Clinical Studies including Therapeutic Trials (ESCISIT)," Annals of the Rheumatic Diseases, vol. 62, pp. 1145-1155, 2003.

[3] D. T. Felson, R. C. Lawrence, M. C. Hochberg et al., "Osteoarthritis: new insights_part 2: treatment approaches," Annals of Internal Medicine, vol. 133, no. 9, pp. 726-737, 2000.

[4] R. W. Moskowitz, S. B. Abramson, and F. Berenbaum, "Coxibs and NSAIDs-clearing the air," Osteoarthritis and Cartilage, vol. 13, no. 7, pp. 545-547, 2005.

[5] J. M. Bjordal, A. E. Ljunggren, A. Klovning, and L. Slordal, "Non-steroidal anti-inflammatory drugs, including cyclooxygenase-2 inhibitors, in osteoarthritis knee: meta-analysis of randomised placebo controlled trials," British Medical Journal, vol. 329, pp. 1317-1322, 2000.

[6] F. M. Arellano, "The withdrawal of rofecoxib," Pharmacoepidemiology and Drug Safety, vol. 14, no. 3, pp. 213-217, 2005.

[7] D. M. Eisenberg, R. B. Davis, S. L. Ettner et al., "Trends in alternative medicine use in the United States, 1990-1997: results of a follow-up national survey," Journal of the American Medical Association, vol. 280, no. 18, pp. 1569-1575, 1998.

[8] J. K. Rao, K. Mihaliak, K. Kroenke, J. Bradley, W. M. Tierney, and M. Weinberger, "Use of complementary therapies for arthritis among patients of rheumatologists," Annals of Internal Medicine, vol. 131, no. 6, pp. 409-416, 1999.

[9] C. J. Herman, P. Allen, W. C. Hunt, A. Prasad, and T. J. Brady, "Use of complementary therapies amoung primary care clinic patients with arthritis," Preventing Chronic Disease, vol. 1, pp. $1-15,2004$.

[10] C. L. Deal and R. W. Moskowittz, "Neutriceuticals as therapeutic agents in osteoarthritis. The role of glucosamine, chondrotin sulphate and collagen hydrolysate," Rheumatic Disease Clinics of North America, vol. 25, pp. 379-395, 1999.

[11] R. Bertken, “"Crystalline DMSO": DMSO2," Arthritis and Rheumatism, vol. 26, no. 5, pp. 693-694, 1983.

[12] K. H. Kolb, G. Janicke, M. Kramer, and P. E. Schulze, "Adsorption, distribution and elimination of labeled DMSO in man and animal," Annals of the New York Academy of Sciences, vol. 141, pp. 85-95, 1967.

[13] L. G. Ameye and W. S. S. Chee, "Osteoarthritis and nutrition. From nutraceuticals to functional foods: a systematic review of the scientific evidence," Arthritis Research and Therapy, vol. 8, no. 4, article R127, 2006.

[14] "Methylsulfonylmethane (MSM). Monograph," Alternative Medicine Review, vol. 8, pp. 438-441, 2003.

[15] J. R. Cronin and K. Ballen, "The biochemistry of alternative therapies: methylsulfonylmethane: nutraceutical of the next century?" Alternative and Complementary Therapies, vol. 5, no. 6, pp. 386-389, 1999.

[16] C. H. Demos, G. L. Beckloff, M. N. Donin, and P. M. Oliver, "Dimethyl sulfoxide in musculoskeletal disorders," Annals of the New York Academy of Sciences, vol. 141, no. 1, pp. 517-523, 1967.

[17] R. A. H. Jimenez and R. F. Willkens, "Dimethyl sulfoxide: a perspective of its use in rheumatic diseases," Journal of Laboratory and Clinical Medicine, vol. 100, no. 4, pp. 489-500, 1982.

[18] J. M. Trice and R. S. Pinals, "Dimethyl sulfoxide: a review of its use in the rheumatic disorders," Seminars in Arthritis and Rheumatism, vol. 15, no. 1, pp. 45-60, 1985.

[19] E. D. Rosenstein, "Topical agents in the treatment of rheumatic disorders," Rheumatic Disease Clinics of North America, vol. 25, no. 4, pp. 899-918, 1999.
[20] A. Ely and B. Lockwood, "What is the evidence for the safety and efficacy of dimethyl sulfoxide and methylsulfonylmethane in pain relief?" Pharmaceutical Journal, vol. 269, no. 7223, pp. 685-687, 2002.

[21] S. Brien, P. Prescott, N. Bashir, H. Lewith, and G. Lewith, "Systematic review of the nutritional supplements dimethyl sulfoxide (DMSO) and methylsulfonylmethane (MSM) in the treatment of osteoarthritis," Osteoarthritis and Cartilage, vol. 16, no. 11, pp. 1277-1288, 2008.

[22] M. S. Evans, K. H. Reid, and J. B. Sharp, "Dimethyl sulphoxide blocks conduction in peripheral nerve C fibres: a possible mode of action," Neuroscience Letters, vol. 150, pp. 145-148, 1993.

[23] M. Muir, "DMSO: many uses, much controversy," Alternative and Complementary Therapies, vol. 2, pp. 230-235, 1996.

[24] S. W. Jacob and R. Herschler, "Dimethyl sulfoxide after twenty years," Annals of the New York Academy of Sciences, vol. 411, pp. 14-18, 1983.

[25] M. A. Beilke, C. Collins-Lech, and P. G. Sohnle, "Effects of dimethyl sulphoxide on the oxidative function of human neutraphils," Journal of Laboratory and Clinical Medicine, vol. 110, pp. 91-96, 1987.

[26] H. E. Paulus, "FDA Arthritis Advisory Committee meeting: methotrexate; guidelines for the clinical evaluation of antiinflammatory drugs; DMSO in scleroderma," Arthritis and Rheumatism, vol. 29, no. 10, pp. 1289-1290, 1986.

[27] I. Muravev, M. Venikova, G. N. Pleslovskaia, T. A. Riazantseva, and I. A. Sigidin, "Effect of dimethylsulphoxide and dimethyl sulfone on a destructive process in the joints of mice with spontaneous arthritis," Patologicheskaia Fiziologiia I Eksperimentalnaia Terapiia, vol. 2, pp. 37-39, 1991.

[28] C. F. Brayton, "Dimethyl sulfoxide (DMSO): a review," The Cornell Veterinarian, vol. 76, no. 1, pp. 61-90, 1986.

[29] K. Ebisuzaki, "Asprin and methylsulphonylmethane (MSM): a search for common mechanisms, with implictions for cancer prevention," Anticancer Research, vol. 23, pp. 453-458, 2003.

[30] S. S. Alam and D. L. Layman, "Dimethyl sulphoxide inhibition of prostacylin production in culture aortic endothelial cells," Annals of the New York Academy of Sciences, vol. 411, pp. 318320, 1983.

[31] R. B. Fox and W. K. Fox, "Dimethyl sulphoxide prevents hydroxyl radical mediated depolymerisation of hyaluronic acid," Annals of the New York Academy of Sciences, vol. 411, pp. 13-17, 1983.

[32] J. Morton and R. Moore, "Lupus nephritis and deaths are diminished in $\mathrm{B} / \mathrm{W}$ mice drinking $3 \%$ water solution of dimethyl sulphoxide or dimethyl sulfone," Journal of Leukocyte Biology, vol. 40, p. 322, 1986.

[33] T. Hasegawa, S. Ueno, S. Kumamoto, and Y. Yoshikai, "Suppressive effect of methylsulfonylmethane (MSM) on type II collagen-induced arthritis in DBA/1J mice," Japanese Pharmacology and Therapeutics, vol. 32, no. 7, pp. 421-427, 2004.

[34] R. Rizzo, M. Grandolfo, C. Godeas, K. W. Jones, and F. Vittur, "Calcium, sulfur, and zinc distribution in normal and arthritic articular equine cartilage: a synchrotron radiation-induced Xray emission (SRIXE) study," Journal of Experimental Zoology, vol. 273, no. 1, pp. 82-86, 1995.

[35] S. Parcell, "Sulfur in human nutrition and applications in medicine," Alternative Medicine Review, vol. 7, no. 1, pp. 2244,2002 . 
[36] A. A. Bookman, S. Williams, and J. Z. Shainhouse, "Effects of a topical diclofenac solution for relieving symptoms of primary osteoarthritis of the knee: a randomized controlled trial," The Canadian Medical Association Journal, vol. 171, pp. 333-338, 2004.

[37] J. H. Brown, "Clinical experience with DMSO in acute musculoskeletal conditions comparing a noncontrolled series with a controlled double blind study," Annals of the New York Academy of Sciences, vol. 141, no. 1, pp. 496-505, 1967.

[38] K. H. Kolb, G. Jaenicke, M. Kramer, and P. E. Schulze, "Absorption, distribution and elimination of labeled dimethyl sulfoxide in man and animals," Annals of the New York Academy of Sciences, vol. 141, no. 1, pp. 85-95, 1967.

[39] U. Vuopala, H. Isomäki, and W. J. Kaipainen, "Dimethyl sulfoxide (DMSO) ointment in the treatment of rheumatoid arthritis. A double blind study," Acta Rheumatologica Scandinavica, vol. 15, no. 2, pp. 139-144, 1969.

[40] Micromedex Health Care Series, Micromedex Inc., Englewood, Colo, USA, 2008, http://lrs.lendac.ie/guides/micromedex/micromedex.html.

[41] Natural Medicine Comprehensive Database, Therapeutic Research Faculty, Stockton, Calif, USA, 5th edition, 2003.

[42] P. R. Usha and M. U. R. Naidu, "Randomised double blind, parallel, placebo controlled study of oral glucosamine, methylsulfonymethane and their combination in osteoarthritis," Clinical Drug Investigation, vol. 24, pp. 353-363, 2004.

[43] L. S. Kim, L. J. Axelrod, P. Howard, N. Buratovich, and R. F. Waters, "Efficacy of methylsulfonylmethane (MSM) in osteoarthritis pain of the knee: a pilot clinical trial," Osteoarthritis and Cartilage, vol. 14, no. 3, pp. 286-294, 2006.

[44] S. Jacobs, R. M. Lawrence, and M. Siegel, Miracle MSM: The Natural Solution for Pain, GP Putnam, New York, NY, USA, 1999.

[45] Patent Storm, September 2008, http://www.patentstorm.us/ patents/6399093/description.html.

[46] B. A. Magnuson, J. Appleton, and G. B. Ames, "Pharmacokinetics and distribution of [35S]methylsulfonylmethane following oral administration to rats," Journal of Agricultural and Food Chemistry, vol. 55, no. 3, pp. 1033-1038, 2007.

[47] S. Otsuki, W. Qian, A. Ishihara, and T. Kabe, "Elucidation of dimethylsulfone metabolism in rat using a ${ }^{35} \mathrm{~S}$ radioisotope tracer method," Nutrition Research, vol. 22, no. 3, pp. 313-322, 2002.

[48] K. Horváth, P. E. Noker, S. Somfai-Relle, R. Glávits, I. Financsek, and A. G. Schauss, "Toxicity of methylsulfonylmethane in rats," Food and Chemical Toxicology, vol. 40, no. 10, pp. 14591462, 2002.

[49] B. A. Magnuson, J. Appleton, B. Ryan, and R. A. Matulka, "Oral developmental toxicity study of methylsulfonylmethane in rats," Food and Chemical Toxicology, vol. 45, no. 6, pp. 977984, 2007.

[50] MSM Guide, September 2008, http://www.msmguide.com/ facts/safety.

[51] A. R. Jadad, R. A. Moore, D. Carroll et al., "Assessing the quality of reports of randomized clinical trials: is blinding necessary?" Controlled Clinical Trials, vol. 17, no. 1, pp. 1-12, 1996.

[52] N. Bellamy, W. W. Buchanan, C. H. Goldsmith, J. Campbell, and L. W. Stitt, "Validation study of WOMAC: a health status instrument for measuring clinically important patient relevant outcomes to antirheumatic drug therapy in patients with osteoarthritis of the hip or knee," Journal of Rheumatology, vol. 15, no. 12, pp. 1833-1840, 1988.
[53] D. Follmann, P. Elliott, I. Suh, and J. Cutler, "Variance imputation for overviews of clinical trials with continuous response," Journal of Clinical Epidemiology, vol. 45, no. 7, pp. 769-773, 1992.

[54] J. Cohen, Statistical Power Analysis for Behavioural Sciences, Lawrence Erlbaum, Hillsdale, NJ, USA, 2nd edition, 1988.

[55] N. J. Koenen, R. F. Haag, P. Bia, and P. Rose, "Perkutane therapie bei aktivierter Gonarthrose," Münchener Medizinische Wochenschrift, vol. 138, pp. 534-538, 1996.

[56] R. Eberhardt, T. Zwingers, and R. Hofmann, "DMSO bei Patienten mit aktivierter Gonathrose. Eine doppeblinde, plazebokontrollierte Phase III Studie," Fortschritte der Medizin, vol. 113, pp. 446-450, 1995.

[57] S. H. Roth and J. Z. Shainhouse, "Efficacy and safety of a topical diclofenac solution (Pennsaid) in the treatment of primary osteoarthritis of the knee: a randomized, doubleblind, vehicle-controlled clinical trial," Archives of Internal Medicine, vol. 164, no. 18, pp. 2017-2023, 2004.

[58] Y. Muravyev, Y. Sigidin, and A. Alyabyeva, "A multicentre study of the effectiveness of dimethyl sulphoxide in rheumatoid and degenerative arthritis," Terapevticheskii Arkhiv, vol. 59, pp. 106-108, 1987.

[59] N. M. R. Thie, N. G. Prasad, and P. W. Major, "Evaluation of glucosamine sulfate compared to ibuprofen for the treatment of temporomandibular joint osteoarthritis: a randomized double blind controlled 3 month clinical trial," Journal of Rheumatology, vol. 28, no. 6, pp. 1347-1355, 2001.

[60] A. M. Wood, I. R. White, and S. G. Thompson, "Are missing outcome data adequately handled? A review of published randomized controlled trials in major medical journals," Clinical Trials, vol. 1, no. 4, pp. 368-376, 2004.

[61] F. Tubach, P. Ravaud, G. Baron et al., "Evaluation of clinically relevant changes in patient reported outcomes in knee and hip osteoarthritis: the minimal clinically important improvement," Annals of the Rheumatic Diseases, vol. 64, no. 1, pp. 2933, 2005.

[62] F. Angst, A. Aeschlimann, and G. Stucki, "Smallest detectable and minimally clinically important differences of rehabilitation interventions with their implications for required sample sizes using WOMAC and SF36 Quality of life measurement instruments in patients with osteoarthritis of the lower extremities," Arthritis Care's Research, vol. 45, pp. 384-391, 2001.

[63] E. W. Ehrich, G. M. Davies, D. J. Watson, J. A. Bolognese, B. C. Seidenberg, and N. Bellamy, "Minimal perceptible clinical improvement with the Western Ontario and McMaster Universities Osteoarthritis Index questionnaire and global assessments in patients with osteoarthritis," Journal of Rheumatology, vol. 27, no. 11, pp. 2635-2641, 2000.

[64] N. Bellamy, J. Kirwan, M. Boers et al., "Recommendations for a core set of outcome measures for future phase III clinical trials in knee, hip, and hand osteoarthritis. Consensus development at OMERACT III," Journal of Rheumatology, vol. 24, no. 4, pp. 799-802, 1997.

[65] P. Brooks, P. Tugwell, C. V. Strand, and L. Simon, "International consensus conference on outcome measures in rheumatology, gold coast Australia, April 11-14 2002," The Journal of Rheumatology, vol. 30, pp. 866-867, 2003.

[66] G. P. Geba, A. L. Weaver, A. B. Polis, M. E. Dixon, and T. J. Schnitzer, "Efficacy of rofecoxib, celecoxib and acetaminophen in osteoarthritis of the knee: a randomised trial," Journal of the American Medical Association, vol. 287, pp. 64-71, 2002. 
[67] E. W. Ehrich, T. J. Schnitzer, H. Mcllwain et al., "Effect of specific COX-2 inhibition in osteoarthritis of the knee: a 6 week double blind, placebp controlled pilot study of rofecoxib.

Rofecoxib Osteoarthritis Pilot Study Group," The Journal of Rheumatology, vol. 26, pp. 2438-2447, 1999. 


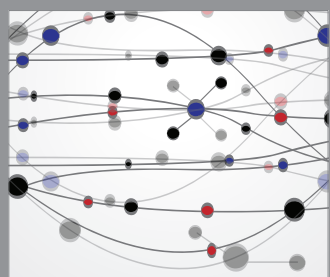

The Scientific World Journal
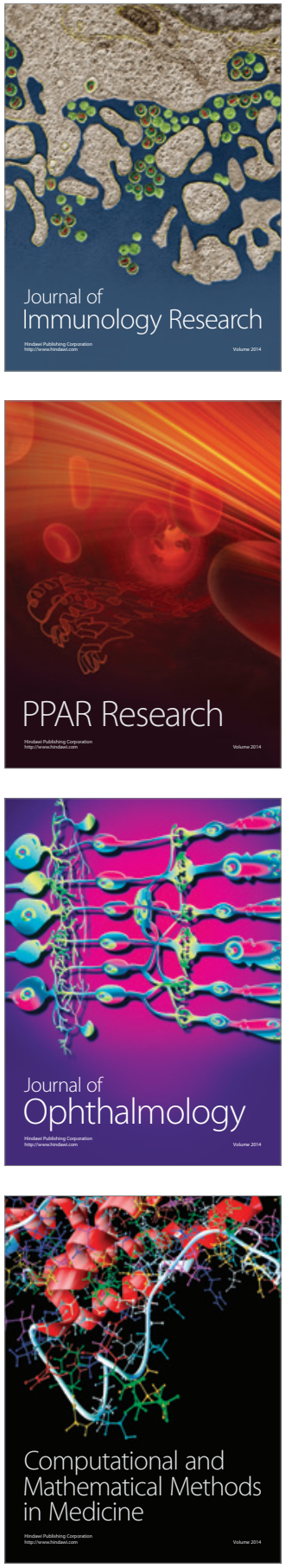

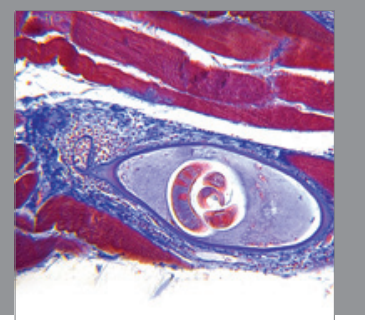

Gastroenterology

Research and Practice
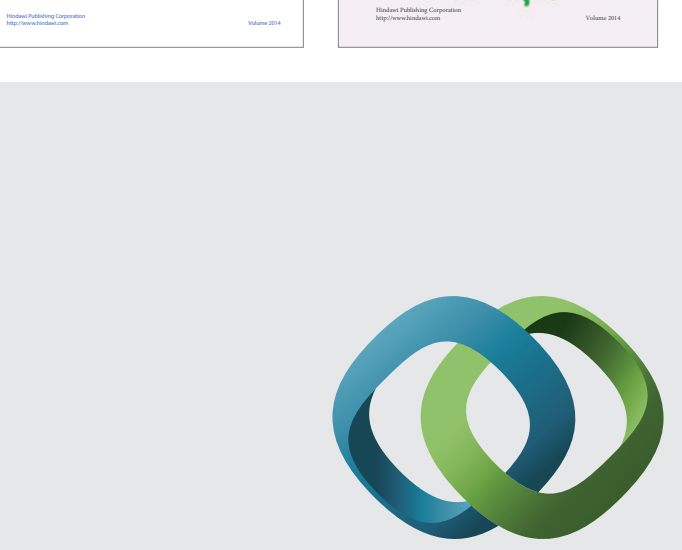

\section{Hindawi}

Submit your manuscripts at

http://www.hindawi.com
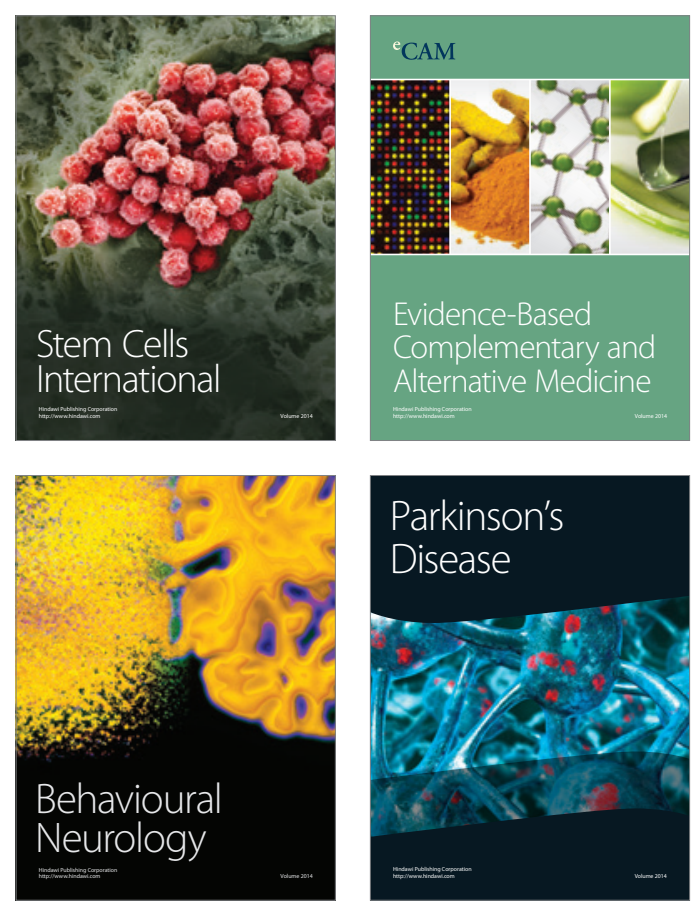

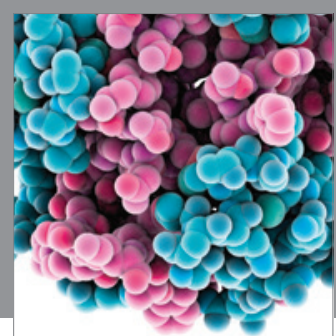

Journal of
Diabetes Research

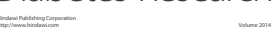

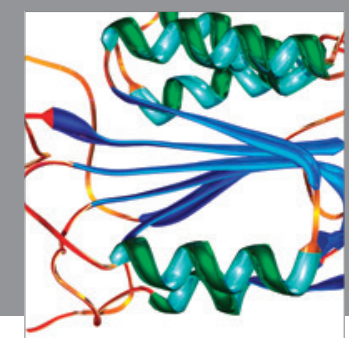

Disease Markers
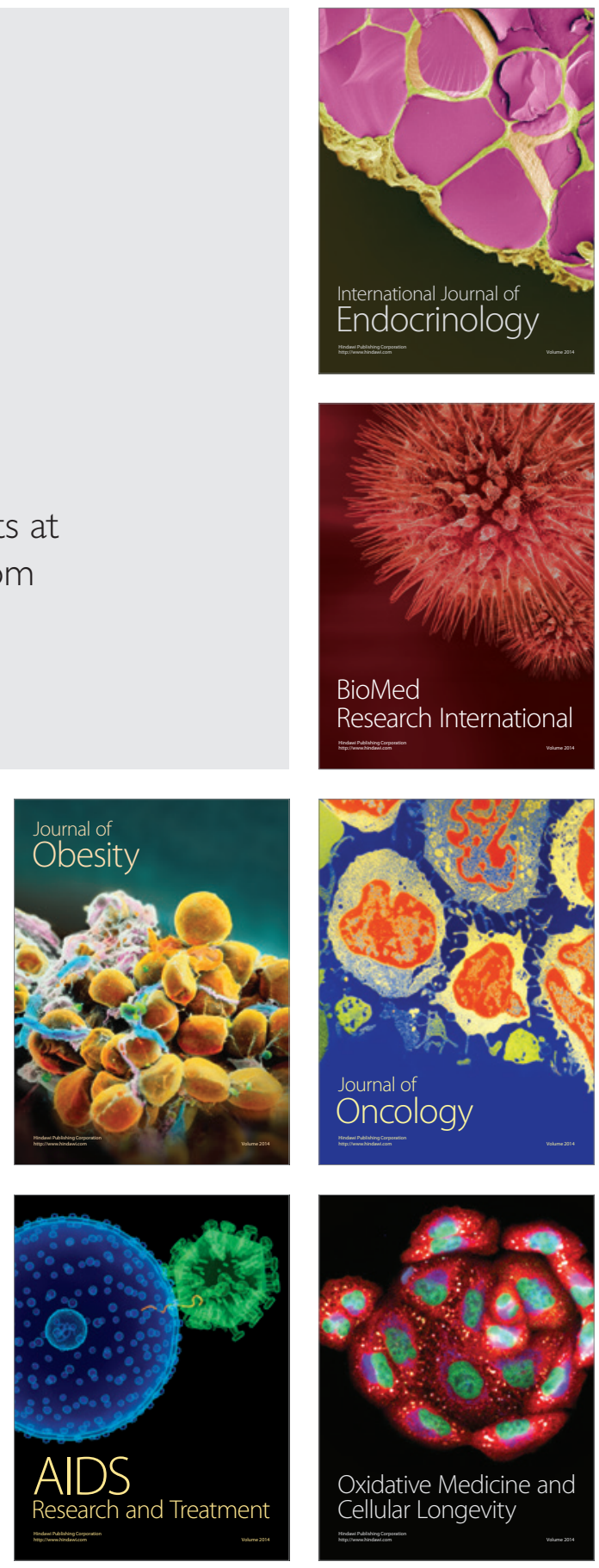